\title{
Why F-ATP Synthase Remains a Strong Candidate as the Mitochondrial Permeability Transition Pore
}

\author{
Paolo Bernardi * \\ Department of Biomedical Sciences, University of Padua, Padua, Italy
}

Keywords: mitochondria, ATP synthase, permeability transition pore, channels, calcium

\section{THE PERMEABILIY TRANSITION AND F-ATP SYNTHASE}

OPEN ACCESS

Edited by:

Miguel A. Aon,

National Institute on Aging (NIA),

United States

Reviewed by:

D. Brian Foster,

Johns Hopkins University,

United States

David F. Stowe,

Medical College of Wisconsin

United States

Ulrich Brandt,

Radboud University Nijmegen Medical

Center, Netherlands

*Correspondence:

Paolo Bernardi

bernardi@bio.unipd.it

Specialty section:

This article was submitted to Mitochondrial Research

a section of the journal

Frontiers in Physiology

Received: 04 September 2018 Accepted: 15 October 2018 Published: 01 November 2018

Citation: Bernardi P (2018) Why F-ATP

Synthase Remains a Strong Candidate as the Mitochondrial

Permeability Transition Pore.

Front. Physiol. 9:1543.

doi: 10.3389/fphys.2018.01543
Mitochondria can undergo a $\mathrm{Ca}^{2+}$-dependent increase of inner membrane permeability called the permeability transition (PT; Hunter et al., 1976). The PT requires $\mathrm{Ca}^{2+}$ accumulation in the matrix and is due to opening of a regulated channel, the PT pore (PTP) which has also been studied by electrophysiology and named mitochondrial megachannel (MMC; Kinnally et al., 1989; Petronilli et al., 1989). The PTP/MMC displays a range of conductance states, which also depend on the species. In mammals maximal conductance can be as high as $1.2 \mathrm{nS}$, which corresponds to a pore with a diameter of about $2-3 \mathrm{~nm}$. The channel is characterized by a variety of lower conductance states, which may allow selective permeation of small solutes (Szabo and Zoratti, 2014). In mammals the PTP is modulated by binding of cyclophilin D, which favors PTP opening. The molecular nature of the PTP/MMC is the matter of active investigation and controversy, and has been specifically addressed in a recent review (Bernardi and Lippe, 2018). The most recent hypothesis posits that it originates from a conformational change occurring on the F-ATP synthase after $\mathrm{Ca}^{2+}$ binding, possibly by replacing $\mathrm{Mg}^{2+}$ at the catalytic site (Giorgio et al., 2017). This proposal has been supported by genetic manipulation of F-ATP synthase (Bonora et al., 2013; Giorgio et al., 2013), by electrophysiological measurements (Giorgio et al., 2013; Alavian et al., 2014; Carraro et al., 2014; von Stockum et al., 2015), and by mutagenesis of specific residues of FATP synthase (Giorgio et al., 2017; Antoniel et al., 2018; Guo et al., 2018; Carraro et al., in press). On the other hand, the Walker laboratory has challenged this hypothesis on the basis of studies where subunit c (He et al., 2017b) or peripheral subunits b and OSCP (He et al., 2017a) had been genetically ablated. These studies are important because they provide the first example of eukaryotic cells where F-ATP synthase subunits have been genetically turned off, thus allowing the first appraisal of the consequences of F-ATP synthase loss of function (He et al., 2017a,b). The Authors also used these cells to address the question of whether the PTP is conserved. They concluded that the PTP was still present in the deletion mutants, and claimed that the idea that FATP synthase is an essential component of the PTP can be ruled out (He et al., 2017a,b). However, analysis of the data suggests that the PTP has been affected by elimination of subunits $c, b$ and OSCP, and that the above conclusion needs to be reassessed.

\section{SIZE MATTERS}

The PTP size is large enough for the diffusion of sucrose, which is the typical solute used to detect occurrence of a PT. Long-lasting PTP opening in vitro is followed by solute diffusion with matrix swelling (Massari and Azzone, 1972). Swelling obviously also occurs in media based on $\mathrm{KCl}$ or other salts, conditions that allow detection of pore(s) of smaller size and/or of lower conductance 
(Ichas et al., 1997). He et al. observed that $\mathrm{Ca}^{2+}$-dependent mitochondrial swelling took place in permeabilized wild-type cells as well as in cells obtained after genetic ablation of subunits $\mathrm{b}(\Delta \mathrm{b})$ and OSCP ( $\triangle$ OSCP) (He et al., 2017a). However, analysis of the data reveals that, compared to wild-type cells, the rate of swelling was reduced to about $20 \%$ in the $\Delta \mathrm{b}$ and to $40 \%$ in the $\triangle$ OSCP cells. This finding may not be immediately appreciated because the ordinate scale of the latter two sets of experiments was expanded (Figure 5 of $\mathrm{He}$ et al., 2017a). The absence of replicates and of calibration with pore-forming agents like alamethicin prevents firm conclusions to be drawn from these experiments. However, decreased swelling rates in $\mathrm{KCl}$ would support the conclusion that in the deletion mutants the PTP size has become smaller, and close to the exclusion size of hydrated $\mathrm{K}^{+}$and $\mathrm{Cl}^{-}$, a difference that would have been even more dramatic in sucrose-based media. In a second set of experiments occurrence of PTP opening was determined based on the $\mathrm{Ca}^{2+}$ load required to trigger $\mathrm{Ca}^{2+}$ release after the accumulation of a train of $\mathrm{Ca}^{2+}$ pulses, the so-called $\mathrm{Ca}^{2+}$ retention capacity (CRC). In these protocols $\mathrm{Ca}^{2+}$ release (which marks onset of the PT) is due to depolarization and does not provide information on the mechanism(s) mediating depolarization itself, which could result even from selective permeabilization to $\mathrm{H}^{+}$as seen in $\mathrm{Ca}^{2+}$ release experiments induced by the addition of protonophores (Bernardi et al., 1984). These experiments are therefore also compatible with opening of a PTP of smaller size.

\section{KNOCK OUT OF F-ATP SYNTHASE SUBUNITS SEVERELY IMPAIRS RESPIRATION}

As a consequence of the ablation of subunits c, b, and OSCP FATP synthase was not properly assembled and rather originated a "vestigial" enzyme that did not display dimer formation after extraction with digitonin. Remarkably, major alterations were also observed in the respiratory chain with almost no mature complex I assembly, severe depletion of complex III and reduction of complex IV. Consistently, respiratory activity was dramatically decreased to between 10 and $20 \%$ of the rate observed in wild-type cells, and could only be marginally stimulated by uncoupler (He et al., 2017a,b). Mitochondrial $\mathrm{Ca}^{2+}$ uptake, which is essential for PTP opening, is driven by the $\mathrm{Ca}^{2+}$ electrochemical gradient $(\Delta \tilde{\mu} \mathrm{Ca}=\mathrm{zF} \Delta \psi+\mathrm{RT} \ln$ $\left.\left[\mathrm{Ca}^{2+}\right]_{\mathrm{i}} /\left[\mathrm{Ca}^{2+}\right]_{\mathrm{o}}\right)$. In respiring mitochondria the driving force for accumulation is the inside-negative membrane potential generated by respiration, and $\mathrm{Ca}^{2+}$ uptake takes place on the uniporter with net charge translocation of 2 (Scarpa and Azzone, 1970; Wingrove et al., 1984; Kirichok et al., 2004). $\mathrm{Ca}^{2+}$ uptake is charge-compensated by increased $\mathrm{H}^{+}$pumping by the respiratory chain, while the buildup of a large $\Delta \mathrm{pH}$ and of high matrix $\mathrm{Ca}^{2+}$ concentration is prevented by $\mathrm{Pi}$ uptake. The essential point for the present discussion is that the maximal rate of $\mathrm{Ca}^{2+}$ uptake is limited by the maximal rate of $\mathrm{H}^{+}$pumping by the respiratory chain (Bragadin et al., 1979), the latter becoming rate-limiting when extramitochondrial $\mathrm{Ca}^{2+}$ rises above about $2 \mu \mathrm{M}$ (Nicholls, 1978). Given that the size of the $\mathrm{Ca}^{2+}$ pulses was $10 \mu \mathrm{M}$ (He et al., 2017a,b) the rate of $\mathrm{Ca}^{2+}$ uptake in the $\Delta c, \Delta b$, and $\triangle$ OSCP mitochondria should have been measurably lower than (rather than identical to) the rate observed in wildtype mitochondria. It is legitimate to ask whether the marked respiratory inhibition described for the deletion mutants is constant over time, or rather compensatory mechanisms exist that eventually restore at least partial expression of F-ATP synthase and of the respiratory chain.

A good example is provided by the $\Delta \mathrm{b}$ mutants. The Authors report the surprising finding that low levels of tryptic peptides corresponding to sequences of subunit $b$ could still be found in the null cells. The peptides were derived from a gel region at $17.5 \mathrm{kDa}$, which corresponds to truncated subunit b. PCR amplification of RNA transcripts covering a putative coding region for these peptides revealed the existence of an alternative splice site in intron A allowing generation of a truncated subunit b (residues 67-214) lacking the membrane region (He et al., 2017a). In summary, the swelling experiments of the Walker laboratory suggest that deletion of the $c$ and peripheral stalk subunits may have affected the PTP, which appears to have become smaller; and contain an internal inconsistency between inhibition of respiration and rates of $\mathrm{Ca}^{2+}$ uptake, which in turn raises questions about the CRC measurements. Thus, these results cannot be used to conclude that the F-ATP synthase does not take part in formation of the PTP.

\section{WHAT WE HAVE LEARNED FROM SITE-DIRECTED MUTAGENESIS}

Several sites have been defined by chemical modification with relatively selective sulfhydryl, histidine and arginine reagents that confer PTP regulation by the membrane potential, matrix $\mathrm{pH}$, divalent cations, quinones, and oxidative stress. Identification of these sites is a formidable challenge but also a unique opportunity to (dis)prove our hypothesis on the identity of the PTP. Indeed, site-directed mutagenesis of specific residues should modify the properties of the PTP in a predictable manner, a task that is made easier by the availability of structures of F-ATP synthase of increasing resolution (Abrahams et al., 1994; Stock et al., 1999; Rubinstein et al., 2003; Strauss et al., 2008; Baker et al., 2012; Davies et al., 2012; Daum et al., 2013; Allegretti et al., 2015; Jiko et al., 2015; Zhou et al., 2015; Hahn et al., 2016; Vinothkumar et al., 2016; Guo et al., 2017).

Residue T163 in the $\beta$ subunit of mammalian F-ATP synthase is essential for the binding of $\mathrm{Mg} / \mathrm{ADP}$ to the catalytic site (Rees et al., 2012). $\mathrm{Mg}^{2+}$ can be replaced by other divalent metals (Selwyn, 1968; Pedersen et al., 1987), and binding of $\mathrm{Ca}^{2+}$ allows ATP hydrolysis without measurable $\mathrm{H}^{+}$pumping (Papageorgiou et al., 1998). In Rhodospirillum rubrum the relative affinity for $\mathrm{Ca}^{2+}$ and $\mathrm{Mg}^{2+}$ could be modulated with a T159S mutation at the $\beta$ subunit (the position equivalent to T163 in mammals), which led to decreased $\mathrm{Ca}^{2+}$-ATPase and increased $\mathrm{Mg}^{2+}$-ATPase activities (Nathanson and Gromet-Elhanan, 2000; Du et al., 2001). We have found that a partial T163S substitution in HeLa cells increases $\mathrm{Mg}^{2+}$-ATP and prevents $\mathrm{Ca}^{2+}$-ATP hydrolysis with a matching decreased sensitivity of the PTP to 
$\mathrm{Ca}^{2+}$, resistance to cell death and decreased apoptosis (Giorgio et al., 2017). One of the most remarkable features of the PTP is its absolute dependence on matrix $\mathrm{Ca}^{2+}$, and these findings are consistent with the idea that the PTP is triggered by $\mathrm{Ca}^{2+}$ binding at the F-ATP synthase catalytic site.

Previous studies had identified a role of Arg residues in the regulation of the PTP through the use of selective reagents such as phenylglyoxal (PGO; Johans et al., 2005). The effects of PGO are species-specific, and we recently identified R107 of FATP synthase subunit $g$ as the unique PTP-modulating target of PGO in yeast. Remarkably, expression of human subunit $\mathrm{g}$ in yeast transferred the "human" PTP phenotype, suggesting that species-specificity depends on differences in the primary structure of F-ATP synthase (Guo et al., 2018). The importance of the $\mathrm{e}$ and $\mathrm{g}$ subunits in formation of the yeast channel is also supported by our recent finding that their deletion abrogated the high-conductance channels in mutants where dimerization was enforced by copper-dependent formation of dimers through oxidation of C23 of subunit a (Carraro et al., in press).

Perhaps the most intriguing feature of the PTP is its inhibition by matrix $\mathrm{H}^{+}$, which is marked at $\mathrm{pH} 6.7$ and leads to complete channel block at pH 6.5 (Nicolli et al., 1993; Antoniel et al., 2018). Based on protection with diethylpyrocarbonate and partial reversal with hydroxylamine, we had concluded that PTP block is mediated by reversible protonation of matrix-accessible His residues (Nicolli et al., 1993). We have recently identified H112 of the OSCP subunit as the unique His responsible for

\section{REFERENCES}

Abrahams, J. P., Leslie, A. G., Lutter, R., and Walker, J. E. (1994). Structure at $2.8 \AA$ resolution of $\mathrm{F}_{1}$-ATPase from bovine heart mitochondria. Nature 370, 621-628. doi: 10.1038/370621a0

Alavian, K. N., Beutner, G., Lazrove, E., Sacchetti, S., Park, H. A., Licznerski, P., et al. (2014). An uncoupling channel within the c-subunit ring of the $\mathrm{F}_{1} \mathrm{~F}_{\mathrm{O}}$ ATP synthase is the mitochondrial permeability transition pore. Proc. Natl. Acad. Sci. U.S.A. 111, 10580-10585. doi: 10.1073/pnas. 1401591111

Allegretti, M., Klusch, N., Mills, D. J., Vonck, J., Kühlbrandt, W., and Davies, K. M. (2015). Horizontal membrane-intrinsic $\alpha$-helices in the stator a-subunit of an F-type ATP synthase. Nature 521, 237-240. doi: 10.1038/nature14185

Antoniel, M., Jones, K., Antonucci, S., Spolaore, B., Fogolari, F., Petronilli, V., et al. (2018). The unique histidine in OSCP subunit of F-ATP synthase mediates inhibition of the permeability transition pore by acidic pH. EMBO Rep. 19, 257-268. doi: 10.15252/embr.201744705

Baker, L. A., Watt, I. N., Runswick, M. J., Walker, J. E., and Rubinstein, J. L. (2012). Arrangement of subunits in intact mammalian mitochondrial ATP synthase determined by cryo-EM. Proc. Natl. Acad. Sci. U.S.A. 109, 11675-11680. doi: 10.1073/pnas.1204935109

Bernardi, P., and Lippe, G. (2018). Channel formation by F-ATP synthase and the permeability transition pore: an update. Curr. Opin. Physiol. 3, 1-5. doi: 10.1016/j.cophys.2017.12.006

Bernardi, P., Paradisi, V., Pozzan, T., and Azzone, G. F. (1984). Pathway for uncoupler-induced calcium efflux in rat liver mitochondria: inhibition by ruthenium red. Biochemistry 23, 1645-1651. doi: 10.1021/bi00303a010

Bonora, M., Bononi, A., De Marchi, E., Giorgi, C., Lebiedzinska, M., Marchi, S., et al. (2013). Role of the $c$ subunit of the $F_{O}$ ATP synthase in mitochondrial permeability transition. Cell Cycle 12, 674-683. doi: 10.4161/cc.23599

Bragadin, M., Pozzan, T., and Azzone, G. F. (1979). Kinetics of $\mathrm{Ca}^{2+}$ carrier in rat liver mitochondria. Biochemistry 18, 5972-5978. doi: 10.1021/bi00593a033 the PTP block by $\mathrm{H}^{+}$. Indeed, $\mathrm{H} 112 \mathrm{Q}$ and $\mathrm{H} 112 \mathrm{Y}$ mutations completely prevented the inhibitory effects of acidic $\mathrm{pH}$ both in PTP-dependent swelling measurements in mitochondria and in single-channel patch-clamp recordings in mitoplasts (Antoniel et al., 2018). Remarkably, the mutations had no consequences on oligomycin-sensitive and uncoupler-stimulated respiration, indicating that the F-ATP synthase was normally assembled.

At variance from knock out experiments, our mutagenesis approach turned out not to be disruptive for F-ATP synthase assembly and catalysis, nor to have detectable effects on respiration and cell viability. We are confident that future work will allow a better understanding of how the energy-conserving enzyme can turn into an energy-dissipating device, a hypothesis that stands and that will be further tested by mutagenesis of Cys residues and by analysis of the channel activity of highly purified preparations from bovine heart.

\section{AUTHOR CONTRIBUTIONS}

The author confirms being the sole contributor of this work and has approved it for publication.

\section{FUNDING}

Research in PB laboratory was supported by Telethon (GGP14037), AIRC (IG17067) and Fondation Leducq (16CVD04).
Carraro, M., Checchetto, V., Sartori, G., Kucharczyk, R., i Rago, J.-P., Minervini, G., et al. (in press). High-conductance channel formation in yeast mitochondria is mediated by F-ATP synthase e and g subunits. Cell. Physiol. Biochem.

Carraro, M., Giorgio, V., Šileikyte, J., Sartori, G., Forte, M., Lippe, G., et al. (2014). Channel formation by yeast F-ATP synthase and the role of dimerization in the mitochondrial permeability transition. j. Biol. Chem. 289, 15980-15985. doi: $10.1074 /$ jbc.C114.559633

Daum, B., Walter, A., Horst, A., Osiewacz, H. D., and Kühlbrandt, W. (2013). Agedependent dissociation of ATP synthase dimers and loss of inner-membrane cristae in mitochondria. Proc. Natl. Acad. Sci. U.S.A. 110, 15301-15306. doi: $10.1073 /$ pnas. 1305462110

Davies, K. M., Anselmi, C., Wittig, I., Faraldo-Gomez, J. D., and Kühlbrandt, W. (2012). Structure of the yeast $F_{1} F_{0}$-ATP synthase dimer and its role in shaping the mitochondrial cristae. Proc. Natl. Acad. Sci. U.S.A. 109, 13602-13607. doi: 10.1073/pnas.1204593109

Du, Z., Tucker, W. C., Richter, M. L., and Gromet-Elhanan, Z. (2001). Assembled $\mathrm{F}_{1}-(\alpha \beta)$ and Hybrid $\mathrm{F}_{1}-\alpha_{3} \beta_{3} \gamma$-ATPases from Rhodospirillum rubrum $\alpha$, wild type or mutant $\beta$, and chloroplast $\gamma$ subunits. Demonstration of $\mathrm{Mg}^{2+}$ versus $\mathrm{Ca}^{2+}$-induced differences in catalytic site structure and function. J. Biol. Chem. 276, 11517-11523. doi: 10.1074/jbc.M007568200

Giorgio, V., Burchell, V., Schiavone, M., Bassot, C., Minervini, G., Petronilli, V., et al. (2017). $\mathrm{Ca}^{2+}$ binding to F-ATP synthase $\beta$ subunit triggers the mitochondrial permeability transition. EMBO Rep. 18, 1065-1076. doi: $10.15252 / \mathrm{embr} .201643354$

Giorgio, V., von Stockum, S., Antoniel, M., Fabbro, A., Fogolari, F., Forte, M., et al. (2013). Dimers of mitochondrial ATP synthase form the permeability transition pore. Proc. Natl. Acad. Sci. U.S.A. 110, 5887-5892. doi: $10.1073 /$ pnas. 1217823110

Guo, H., Bueler, S. A., and Rubinstein, J. L. (2017). Atomic model for the dimeric $\mathrm{F}_{\mathrm{O}}$ region of mitochondrial ATP synthase. Science 358, 936-940. doi: $10.1126 /$ science.aao 4815 
Guo, L., Carraro, M., Sartori, G., Minervini, G., Eriksson, O., Petronilli, V., et al. (2018). Arginine 107 of yeast ATP synthase subunit g mediates sensitivity of the mitochondrial permeability transition to phenylglyoxal. J. Biol. Chem. 293, 14632-14645. doi: 10.1074/jbc.RA118.004495

Hahn, A., Parey, K., Bublitz, M., Mills, D. J., Zickermann, V., Vonck, J., et al. (2016). Structure of a complete ATP synthase dimer reveals the molecular basis of inner mitochondrial membrane morphology. Mol. Cell 63, 445-456. doi: 10.1016/j.molcel.2016.05.037

He, J., Carroll, J., Ding, S., Fearnley, I. M., and Walker, J. E. (2017a). Permeability transition in human mitochondria persists in the absence of peripheral stalk subunits of ATP synthase. Proc. Natl. Acad. Sci. U.S.A. 114, 9086-9091. doi: $10.1073 /$ pnas. 1711201114

He, J., Ford, H. C., Carroll, J., Ding, S., Fearnley, I. M., and Walker, J. E. (2017b). Persistence of the mitochondrial permeability transition in the absence of subunit c of human ATP synthase. Proc. Natl. Acad. Sci. U.S.A. 114, 3409-3414. doi: $10.1073 /$ pnas. 1702357114

Hunter, D. R., Haworth, R. A., and Southard, J. H. (1976). Relationship between configuration, function, and permeability in calcium-treated mitochondria. J. Biol. Chem. 251, 5069-5077.

Ichas, F., Jouaville, L. S., and Mazat, J. P. (1997). Mitochondria are excitable organelles capable of generating and conveying electrical and calcium signals. Cell 89, 1145-1153. doi: 10.1016/S0092-8674(00)80301-3

Jiko, C., Davies, K. M., Shinzawa-Itoh, K., Tani, K., Maeda, S., Mills, D. J., et al. (2015). Bovine F1Fo ATP synthase monomers bend the lipid bilayer in 2D membrane crystals. Elife Sci. 4:e06119. doi: 10.7554/eLife.06119

Johans, M., Milanesi, E., Franck, M., Johans, C., Liobikas, J., Panagiotaki, M., et al. (2005). Modification of permeability transition pore arginine(s) by phenylglyoxal derivatives in isolated mitochondria and mammalian cells: structure-function relationship of arginine ligands. J. Biol. Chem. 280, 12130-12136. doi: 10.1074/jbc.M413454200

Kinnally, K. W., Campo, M. L., and Tedeschi, H. (1989). Mitochondrial channel activity studied by patch-clamping mitoplasts. J. Bioenerg. Biomembr. 21, 497-506. doi: 10.1007/BF00762521

Kirichok, Y., Krapivinsky, G., and Clapham, D. E. (2004). The mitochondrial calcium uniporter is a highly selective ion channel. Nature 427, 360-364. doi: 10.1038 /nature02246

Massari, S., and Azzone, G. F. (1972). The equivalent pore radius of intact and damaged mitochondria and the mechanism of active shrinkage. Biochim. Biophys. Acta 283, 23-29. doi: 10.1016/0005-2728(72)90094-1

Nathanson, L., and Gromet-Elhanan, Z. (2000). Mutations in the beta-subunit $\mathrm{Thr}^{159}$ and $\mathrm{Glu}^{184}$ of the Rhodospirillum rubrum $\mathrm{F}_{0} \mathrm{~F}_{1}$ ATP synthase reveal differences in ligands for the coupled $\mathrm{Mg}^{2+}$ - and decoupled $\mathrm{Ca}^{2+}$-dependent $\mathrm{F}_{0} \mathrm{~F}_{1}$ activities. J. Biol. Chem. 275, 901-905. doi: 10.1074/jbc.275.2.901

Nicholls, D. G. (1978). The regulation of extramitochondrial free calcium ion concentration by rat liver mitochondria. Biochem. J. 176, 463-474. doi: 10.1042/bj1760463

Nicolli, A., Petronilli, V., and Bernardi, P. (1993). Modulation of the mitochondrial cyclosporin A-sensitive permeability transition pore by matrix $\mathrm{pH}$. Evidence that the pore open-closed probability is regulated by reversible histidine protonation. Biochemistry 32, 4461-4465. doi: 10.1021/bi00067a039

Papageorgiou, S., Melandri, A. B., and Solaini, G. (1998). Relevance of divalent cations to ATP-driven proton pumping in beef heart mitochondrial $\mathrm{F}_{0} \mathrm{~F}_{1}$ ATPase. J. Bioenerg. Biomembr. 30, 533-541. doi: 10.1023/A:1020528432609
Pedersen, P. L., Williams, N., and Hullihen, J. (1987). Mitochondrial ATP synthase: dramatic $\mathrm{Mg}^{2+}$-induced alterations in the structure and function of the F1-ATPase moiety. Biochemistry 26, 8631-8637. doi: 10.1021/ bi00400a021

Petronilli, V., Szabó, I., and Zoratti, M. (1989). The inner mitochondrial membrane contains ion-conducting channels similar to those found in bacteria. FEBS Lett. 259, 137-143. doi: 10.1016/0014-5793(89)81513-3

Rees, D. M., Montgomery, M. G., Leslie, A. G., and Walker, J. E. (2012). Structural evidence of a new catalytic intermediate in the pathway of ATP hydrolysis by $\mathrm{F}_{1}$-ATPase from bovine heart mitochondria. Proc. Natl. Acad. Sci. U.S.A. 109, 11139-11143. doi: 10.1073/pnas.1207587109

Rubinstein, J. L., Walker, J. E., and Henderson, R. (2003). Structure of the mitochondrial ATP synthase by electron cryomicroscopy. EMBO J. 22, 6182-6192. doi: 10.1093/emboj/cdg608

Scarpa, A., and Azzone, G. F. (1970). The mechanism of ion translocation in mitochondria. 4. Coupling of $\mathrm{K}^{+}$efflux with $\mathrm{Ca}^{2+}$ uptake. Eur. J. Biochem. 12, 328-335. doi: 10.1111/j.1432-1033.1970.tb00854.x

Selwyn, M. J. (1968). Model reaction for mitochondrial adenosine triphosphatase. Nature 219, 490-493. doi: 10.1038/219490a0

Stock, D., Leslie, A. G., and Walker, J. E. (1999). Molecular architecture of the rotary motor in ATP synthase. Science 286, 1700-1705. doi: $10.1126 /$ science. 286.5445 .1700

Strauss, M., Hofhaus, G., Schröder, R. R., and Kühlbrandt, W. (2008). Dimer ribbons of ATP synthase shape the inner mitochondrial membrane. EMBO J. 27, 1154-1160. doi: 10.1038/emboj.2008.35

Szabo, I., and Zoratti, M. (2014). Mitochondrial channels: ion fluxes and more. Physiol. Rev. 94, 519-608. doi: 10.1152/physrev.00021.2013

Vinothkumar, K. R., Montgomery, M. G., Liu, S., and Walker, J. E. (2016). Structure of the mitochondrial ATP synthase from Pichia angusta determined by electron cryo-microscopy. Proc. Natl. Acad. Sci. U.S.A. 113, 12709-12714. doi: 10.1073/pnas.1615902113

von Stockum, S., Giorgio, V., Trevisan, E., Lippe, G., Glick, G. D., Forte, M. A., et al (2015). F-ATPase of D. melanogaster forms 53 Picosiemen (53-pS) channels responsible for mitochondrial $\mathrm{Ca}^{2+}$-induced $\mathrm{Ca}^{2+}$ release. J. Biol. Chem. 290, 4537-4544. doi: 10.1074/jbc.C114.629766

Wingrove, D. E., Amatruda, J. M., and Gunter, T. E. (1984). Glucagon effects on the membrane potential and calcium uptake rate of rat liver mitochondria. J. Biol. Chem. 259, 9390-9394.

Zhou, A., Rohou, A., Schep, D. G., Bason, J. V., Montgomery, M. G., Walker, J. E., et al. (2015). Structure and conformational states of the bovine mitochondrial ATP synthase by cryo-EM. Elife Sci. 4:e10180. doi: 10.7554/eLife. 10180

Conflict of Interest Statement: The author declares that the research was conducted in the absence of any commercial or financial relationships that could be construed as a potential conflict of interest.

Copyright (c) 2018 Bernardi. This is an open-access article distributed under the terms of the Creative Commons Attribution License (CC BY). The use, distribution or reproduction in other forums is permitted, provided the original author(s) and the copyright owner(s) are credited and that the original publication in this journal is cited, in accordance with accepted academic practice. No use, distribution or reproduction is permitted which does not comply with these terms. 